Research Article

\title{
Structural Design and Mechanical Performance Analysis of Carbon Fiber Closed Fixtures for UHV Transmission Lines
}

\author{
Yujiao Zhang, ${ }^{1}$ Cheng Yan, ${ }^{2}$ Xiongfeng Huang ${ }^{(D)},{ }^{1}$ and Yanran Chen ${ }^{3}$ \\ ${ }^{1}$ School of Electrical Engineering and Automation, Hefei University of Technology, Hefei 230009, China \\ ${ }^{2}$ State Grid Zhangzhou Power Supply Company, Zhangzhou 363000, China \\ ${ }^{3}$ State Grid Yichang Power Supply Company, Yichang 443000, China \\ Correspondence should be addressed to Xiongfeng Huang; sxdxhuangxf@163.com
}

Received 6 August 2020; Revised 16 December 2020; Accepted 19 January 2021; Published 2 February 2021

Academic Editor: Guido Ala

Copyright (c) 2021 Yujiao Zhang et al. This is an open access article distributed under the Creative Commons Attribution License, which permits unrestricted use, distribution, and reproduction in any medium, provided the original work is properly cited.

\begin{abstract}
The closed clamp is a standard tool for the insulator replacement in ultrahigh voltage (UHV) transmission lines, which is mainly made of titanium alloy material and weighs more than $27 \mathrm{~kg}$ that greatly reduces the working efficiency for operators. Due to the lightweight demand, carbon fiber composite materials are applied to design a new type of clamp, in which mechanical properties of new fixtures need to be fulfilled while considering poor impact resistance and low interlaminar shear strength of carbon fiber composite materials. To excavate a high-strength ply structure, finite element progressive damage strength analysis is employed to evaluate the mechanical properties of three different ply angles of the carbon fiber closed fixture, in which Tsai-Wu strength theory is thought as the strength judgment basis for carbon fiber composite materials. After comparison with the displacement-load curves, the three different ply angles fail to meet the strength requirements. So, a carbon fiber laminate structure with an outer cladding for carbon fiber closed fixtures is raised and verified. The analysis results show that the laminate structure meets the strength requirements. Destructive test of the new closed clamp is conducted to verify the correctness of the proposed method, and the weight is reduced by $36.46 \%$.
\end{abstract}

\section{Introduction}

The closed fixture is the most commonly used tool for replacing the porcelain or glass insulators in ultrahigh voltage transmission lines, which consists of a front fixture, a rear fixture, and a screw, as shown in Figure 1. Currently, closed clamps are mainly made of titanium alloy materials $[1,2]$. Because of large size and large load of the insulators, the weight of titanium alloy closed clamps has increased to about $27 \mathrm{~kg}$ with the strength requirements. For the workers, it is extremely inconvenient to operate, which reduces the efficiency of insulator replacement. Therefore, lightweight demand is aroused for the design of the closed fixture.

Carbon fiber composite materials are light materials, which are fit for reducing the weight of closed clamp, because the density of carbon fiber materials is 0.38 times that of titanium alloy materials, and the strength is more than 5 times that of titanium alloy materials [3]. But considering poor impact resistance and low interlaminar shear strength
$[4,5]$, carbon fiber material is difficult to achieve the required mechanical properties for large load-bearing components like UHV closed clamps.

As carbon fiber composites are the same as polymer matrix composites, different thickness and mechanical properties (Young's modulus and strain energy release rate) have great influence on the mechanical properties of the composites $[6,7]$. Therefore, in order to meet the mechanical performance requirements, carbon fiber laminate structures need to be elaborated. A quasi-isotropic pavement structure with $0^{\circ}, \pm 45^{\circ}$, and $90^{\circ}$ cross-arrangement is proposed to meet the mechanical performance requirements of some bent and sheared parts [8-11]. And to reduce the weight and improve the flexural rigidity and the stability, a carbon fiber foam sandwich structure is discussed in the aerospace [12-15]. For this sandwich structure, the upper and lower panels mainly bear in-plane tensile and compression, and in-plane shear loads, while the core bears compression and shear loads. Although these pavement structures meet the mechanical performance 


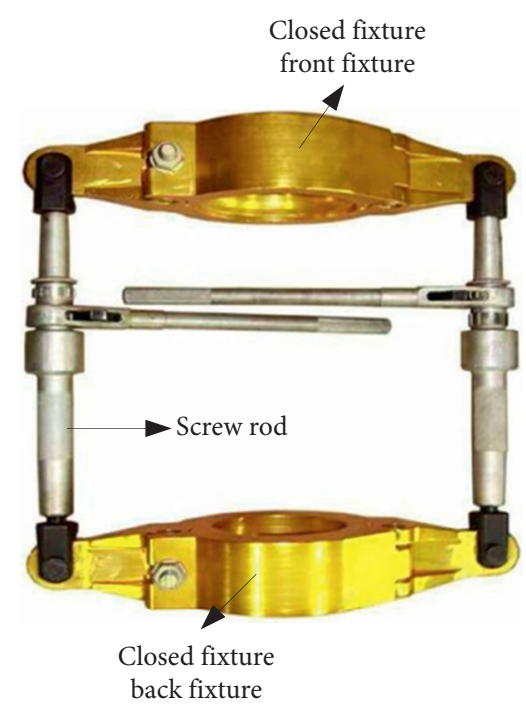

FIgURE 1: Titanium alloy closed fixture.

requirements, they are only used on small load-bearing components. For the big load-bearing closed fixtures which require a weight of less than $20 \mathrm{~kg}$ and a strength of more than $200 \mathrm{kN}$, this type of application is scarce, so a new layer structure needs to be exploited for the carbon fiber closed fixture. To assess the strength of carbon fiber members quickly and accurately, the progressive damage strength analysis method is employed [16-18] as the finite element strength analysis method of carbon fiber members, and Tsai-Wu strength theory is preferred as the strength judgment. At present, the most commonly used strength theories are Hoffman strength theory, Tsai-Hill strength theory, and Tsai-Wu strength theory [19-21]. Usually, Tsai-Wu strength theory is thought as a higher accuracy and more comprehensive description of materials than other theories.

In this paper, a novel carbon fiber laminate structure with an outer cladding for carbon fiber closed fixtures is proposed for the design of new $1000 \mathrm{kV}$ ultrahigh voltage closed fixture. Compared with the structure of the traditional titanium alloy closed fixture, the structure of the carbon fiber closed fixture is raised considering the enhancement of the shear and bending resistance at the ends of the two wings of the fixture. To protect the carbon fiber from being damaged and improve the bending resistance and shear strength of the new fixture, this paper firstly designs a new shape for the carbon fiber closed fixture and proposes three traditional bending and shear strength ply structures. To compare and analyze the mechanical properties of the three types, the finite element progressive damage strength analysis method is employed, in which Tsai-Wu tensor theory is thought as the strength judgment basis for carbon fiber composite materials. Then, on the basis of the three traditional layup structures, a novel layup structure with an outer cladding is designed for the fixture to further increase the strength. At last, the mechanical test is to be conducted by the tensile machine for the verification of the feasibility of the structural design and the effectiveness of the finite element calculation.

\section{Structural Design of the Carbon Fiber Closed Fixture}

2.1. Shape Structure of the Traditional Titanium Alloy Closed Fixture. To design the new structure of the $1000 \mathrm{kV}$ closed fixture based on carbon fiber materials, the XWP-420 closed fixture made of titanium alloy material is selected as a reference, in which the rated load is $80 \mathrm{kN}$, the safety factor is 2.5 with $200 \mathrm{kN}$ maximum load, and the total weight is $27.48 \mathrm{~kg}$. The outline structure of XWP-420 is shown in Figure 2. The difference between the front fixture and the rear fixture is only the structure of the inner cavity, and the rest are the same.

When replacing the insulator, the front clamp and the rear clamp are, respectively, clamped on the front and rear insulator steel caps. And then the screw rod is tightened to transfer the load on the insulator to the clamp. Finally, the operator removes the insulator and replaces it with a new one, as shown in Figure 3(a).

While tightening the screw rod of the closed fixture, the contact part between the closed fixture and the steel cap is regarded as fixed constraint and the end of the left and right wings is regarded as the loading end [1]. And during the analysis of the mechanical properties, the front fixture and the rear fixture are regarded as a simple beam balanced by three points of force shown in Figure 3(b), considering that the forces and dimensions of the front and rear fixtures are exactly the same.

According to the loading characteristics and boundary conditions of the fixture, the structure mainly bears the bending moment and shear force. The overall structure is simplified as a simply supported cantilever beam.

2.2. Concept of Exterior Design. According to the working condition of the closed fixture, since the inner cavity wall of the closed fixture directly contacts the insulator steel cap, the inner cavity wall will be rubbed by the steel cap when the screw is tightened. Therefore, considering that carbon fiber is a non-wear-resistant material, a layer of metal foil is glued to the inner cavity wall of the carbon fiber closed fixture. According to Figure 3, the closed fixture needs to make bolt holes at the ends of both wings for connecting the screw rods. If bolt holes are drilled at the ends of the two wings of the carbon fiber closed fixture, the fiber continuity of the carbon fiber will be destroyed and the strength of the fixture will be reduced. Therefore, this article designed a metal sleeve with bolt holes, glued to the ends of the two wings of the carbon fiber closed clamp. The new carbon fiber closed clamp is shown in Figure 4; the sheet metal and the metal sleeve are made of aluminum alloy.

Considering the higher brittleness of new materials and the safety factor set in XWP-420 closed fixture, the safety factor of carbon fiber closed fixture is set to 3 ; that is, the maximum load is allowed as $240 \mathrm{kN}$. To improve the bending resistance and shear resistance of the fixture, the thickness of the fixture and the height of the ends of the two wings are increased. And according to the external dimensions of the $420 \mathrm{kN}$ ceramic insulator steel cap and the 


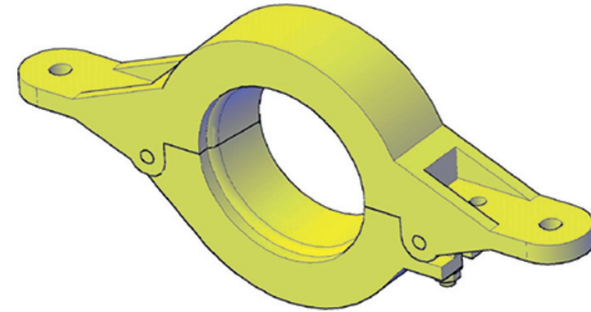

(a)

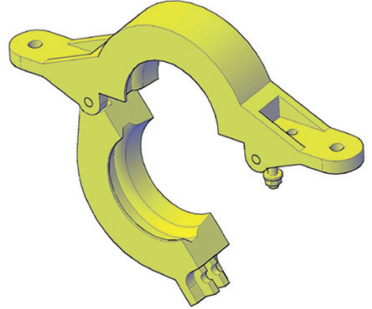

(b)

Figure 2: Schematic diagram of XWP-420 closed fixture: (a) front fixture; (b) rear fixture.

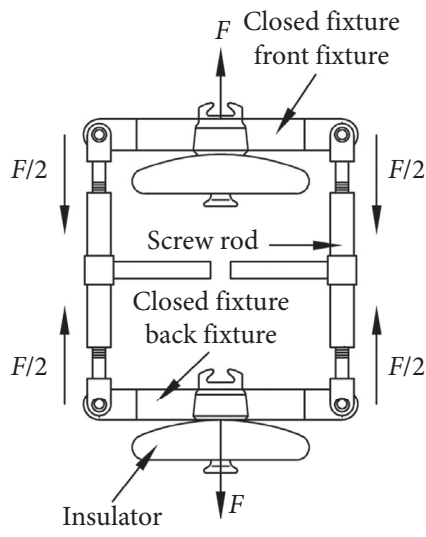

(a)

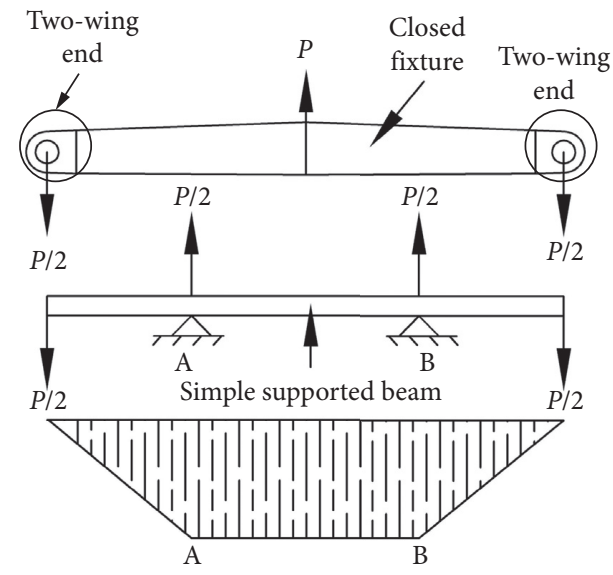

(b)

Figure 3: Analysis of the stress characteristics of the closed fixture: (a) closed fixture work diagram; (b) closed fixture force diagram.

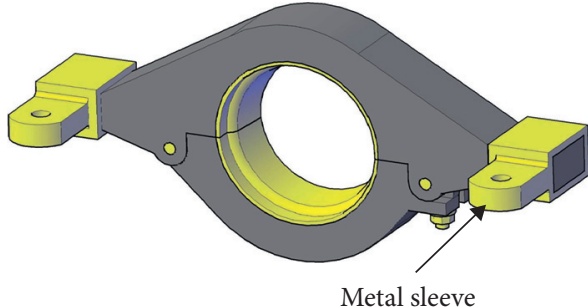

(a)

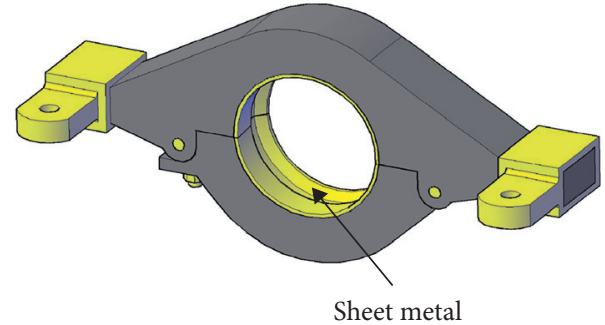

(b)

FiguRE 4: Structural chart of the carbon fiber closed fixture: (a) carbon fiber closed fixture front fixture; (b) carbon fiber closed fixture rear fixture.

size of the umbrella skirt, the external dimensions of the ultrahigh voltage carbon fiber closed clamp are designed. Dimensions of various components of the carbon fiber closed clamp are shown in Figure 5.

2.3. Structural Design of Pavement. T700/T009 type carbon fiber composite material is used to fabricate the clamp. The mechanical parameters of the material are shown in Table 1.
Fiber directions of the carbon fiber laminate are shown in Figure 6. Direction 1 indicates the fiber direction, direction 2 indicates the direction perpendicular to the fiber direction in the paving layer, and direction 3 indicates the paving layer direction.

According to the classic laminate theory [22], the relationship between the internal force and strain of the laminate and the internal torque and strain of the laminate is expressed as follows: 


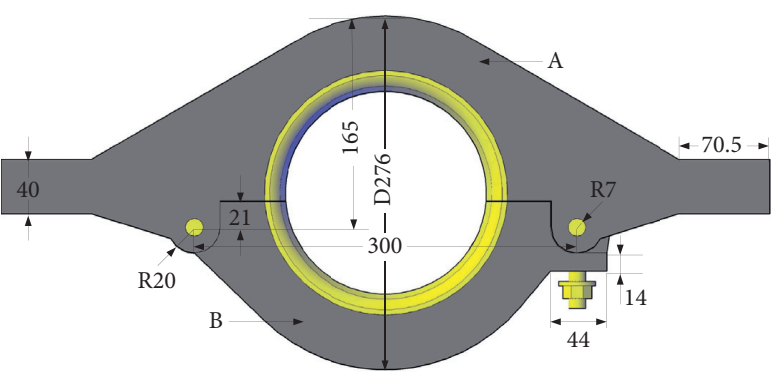

(a)

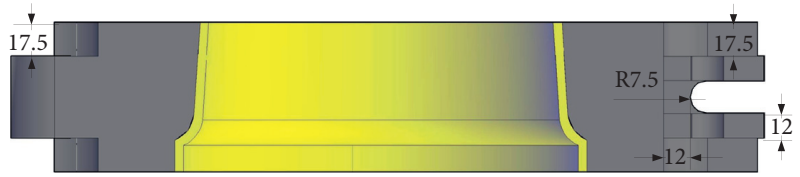

(c)

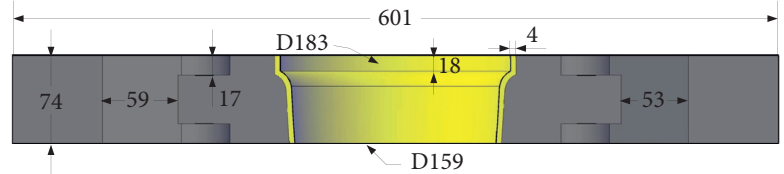

(b)

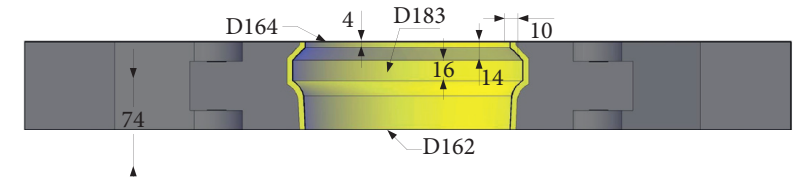

(d)

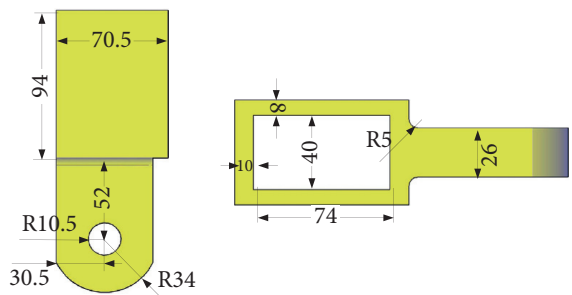

(e)

Figure 5: Dimensions of various components of the carbon fiber closed clamp (unit: $\mathrm{mm}$ ): (a) carbon fiber front fixture overall structure, (b) A part bottom view, (c) B part top view, (d) structural dimension of the inner cavity of the carbon fiber rear fixture, and (e) dimensions of the metal sleeve outline.

TABle 1: Mechanical parameters of T700/T009 material.

\begin{tabular}{lccc}
\hline Direction & Elastic modulus & Tensile strength & Compressive strength \\
\hline Direction 1 $(\mathrm{MPa})$ & 140000 & 2616 & 965 \\
Direction 2 $(\mathrm{MPa})$ & 9000 & 47 & 168 \\
Direction 3 $(\mathrm{MPa})$ & 9000 & 47 & 124 \\
Direction & Shear modulus & Shear strength & 72 \\
$1-2$ face $(\mathrm{MPa})$ & 4700 & 51 & Poisson's ratio \\
$2-3$ face $(\mathrm{MPa})$ & 3100 & 71 & 0.32 \\
$1-3$ face $(\mathrm{MPa})$ & 4700 & Single-layer thickness $/ \mathrm{mm}$ & 0.45 \\
Density $\left(\mathrm{g} / \mathrm{cm}^{3}\right)$ & 1.61 & 0.32 \\
\hline
\end{tabular}

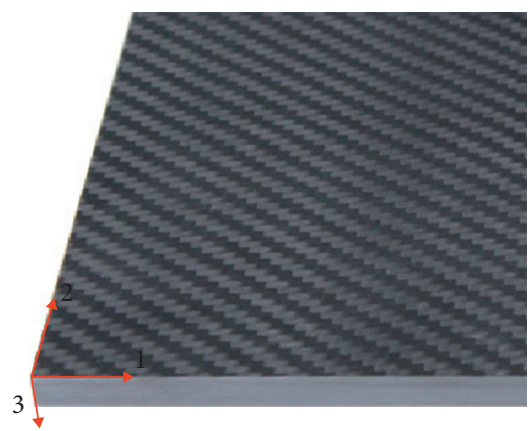

Figure 6: Fiber direction of the carbon fiber laminate. 


$$
\begin{aligned}
& \left\{\begin{array}{c}
T_{x} \\
T_{y} \\
T_{x y}
\end{array}\right\}=\left[\begin{array}{lll}
B_{11} & B_{12} & B_{16} \\
B_{12} & B_{22} & B_{26} \\
B_{16} & B_{26} & B_{66}
\end{array}\right]\left\{\begin{array}{c}
\varepsilon_{x}^{0} \\
\varepsilon_{y}^{0} \\
\gamma_{x y}^{0}
\end{array}\right\}+\left[\begin{array}{lll}
D_{11} & D_{12} & D_{16} \\
D_{12} & D_{22} & D_{26} \\
D_{16} & D_{26} & D_{66}
\end{array}\right]\left\{\begin{array}{c}
k_{x} \\
k_{y} \\
k_{x y}
\end{array}\right\}, \\
& \left\{\begin{array}{c}
T_{x} \\
T_{y} \\
T_{x y}
\end{array}\right\}=\left[\begin{array}{lll}
B_{11} & B_{12} & B_{16} \\
B_{12} & B_{22} & B_{26} \\
B_{16} & B_{26} & B_{66}
\end{array}\right]\left\{\begin{array}{c}
\varepsilon_{x}^{0} \\
\varepsilon_{y}^{0} \\
\gamma_{x y}^{0}
\end{array}\right\}+\left[\begin{array}{lll}
D_{11} & D_{12} & D_{16} \\
D_{12} & D_{22} & D_{26} \\
D_{16} & D_{26} & D_{66}
\end{array}\right]\left\{\begin{array}{c}
k_{x} \\
k_{y} \\
k_{x y}
\end{array}\right\},
\end{aligned}
$$

where $F_{x}, F_{y}$, and $F_{x y}$ denote the internal forces per unit width (or length) on the cross section of the laminated plate; $T_{x}, T_{y}$, and $T_{x y}$ denote the internal moments per unit width (or length) on the cross section of the laminated plate; and $\varepsilon_{x}^{o}, \varepsilon_{y}^{o}$, and $\gamma_{x y}^{o}$ are the midplane strain. $k_{x}, k_{y}$, and $k_{x y}$ are the curvature and twist rate of the middle plane. $A_{i j}$ denotes the tensile stiffness, $B_{i j}$ denotes the coupling stiffness, and $D_{i j}$ denotes the bending stiffness; $i$ and $j$ belong to the collection of positive integer.

According to the calculation formula of coupling stiffness,

$$
\begin{aligned}
& B_{i j}=\sum_{k=1}^{n} \frac{\left(\bar{Q}_{i j}\right)_{k}\left(Z_{k}^{2}-Z_{k-1}^{2}\right)}{2}=\frac{\left(\bar{Q}_{i j}\right)_{1}\left(Z_{1}^{2}-Z_{0}^{2}\right)}{2}+ \\
& \frac{\left(\bar{Q}_{i j}\right)_{2}\left(Z_{2}^{2}-Z_{1}^{2}\right)}{2}+\cdots+\frac{\left(\bar{Q}_{i j}\right)_{n / 2}\left(0-Z_{n / 2}^{2}-1\right)}{2}+ \\
& \frac{\left(\bar{Q}_{i j}\right)_{n / 2+1}\left(Z_{n / 2+1}^{2}\right)}{2}+\cdots+\frac{\left(\bar{Q}_{i j}\right)_{n-1}\left(Z_{n-1}^{2}-Z_{n-2}^{2}\right)}{2}+ \\
& \frac{\left(\bar{Q}_{i j}\right)_{n}\left(Z_{n}^{2}-Z_{n-1}^{2}\right)}{2},
\end{aligned}
$$

where $\bar{Q}_{i j}$ represents the reduction stiffness coefficient and $Z$ represents the distance from one layer of the laminate to the middle of the laminate.

For carbon fiber laminates with general layup methods, $\left(\bar{Q}_{i j}\right)_{1} \neq\left(\bar{Q}_{i j}\right)_{n}$ and $\left(Z_{1}^{2}-Z_{0}^{2}\right) \neq-\left(Z_{n}^{2}-Z_{n-1}^{2}\right)$. The coupling stiffness is not equal to zero, which produces the coupling of tensile force and shear force, tensile force, and bending moment, that affects the rigidity and strength of the entire laminate. However, for carbon fiber laminates with symmetrical layup, $\left(\bar{Q}_{i j}\right)_{1}=\left(\bar{Q}_{i j}\right)_{n}$ and $\left(Z_{1}^{2}-Z_{0}^{2}\right)=-\left(Z_{n}^{2}-\right.$ $\left.Z_{n-1}^{2}\right)$. The coupling stiffness is equal to zero, so there is no coupling between tensile force and shear force, tensile force and bending moment, that is conducive to enhancing the rigidity and strength of the laminate. Therefore, a symmetrical layup method is adopted for the design of the carbon fiber closed fixtures.

At present, for the selection of the ply angle, $0^{\circ}, 90^{\circ}$, and $\pm 45^{\circ}$ are generally preferred. The special angle process of $\pi / 4$ is easy to operate and is more accurate to be grasped and controlled, which improves the in-plane shear stiffness and strength of carbon fiber laminates [23].

According to the thickness of the new closed fixture, three kinds of layup angles are investigated as shown in Table 2 .

\section{Method for Analyzing Mechanical Properties of the Carbon Fiber Closed Fixture}

The progressive damage strength analysis method is used to analyze the mechanical properties of the fixture, which mainly completes the calculation through three parts such as stress analysis, failure analysis, and rigidity reduction.

3.1. Progressive Damage Strength Analysis Method. The progressive damage strength analysis method is a calculation method based on the assumption that a certain bearing capacity is retained after material damage [16], where approximate processes are shown as Figure 7.

Based on the Finite Element Modeling (FEM) models for the component, stress analysis is conducted first, and then the termination calculation is determined. The termination calculation conditions are as follows. (1) Calculate the applied displacement load to reach the set expected displacement load. (2) The entire layer of fibers in a certain layer of carbon fiber laminate is damaged and damaged, resulting in the failure of the entire carbon fiber laminate.

If the termination calculation condition is fulfilled, the calculation is exited, the progressive damage strength analysis is completed, and otherwise, the damage analysis is performed. During the failure analysis, the stress of the element obtained by the stress analysis is brought into the appropriate strength criterion to determine whether the element is damaged.

If the element is not damaged, the load is applied. And then a new round of calculation is started. If the element is broken, the stiffness is reduced first, then the load is applied, and then a new round of calculation is started. During this cycle, until the termination calculation condition is satisfied, the progressive damage strength analysis is completed.

3.2. Strength Criterion of the Carbon Fiber Composite. In failure analysis, strength criterion is required. For the judgment of the destruction of the components made by carbon fiber materials, Tsai-Wu strength theory is involved considering that a comprehensive description of material failure is specified compared to other strength theories and is closer to the experimental value [19]. [20]:

The expression of Tsai-Wu strength theory is as follows

$$
\begin{aligned}
& W=F_{1} \sigma_{1}+F_{2} \sigma_{2}+F_{3} \sigma_{3}+F_{11} \sigma_{1}^{2}+F_{22} \sigma_{2}^{2}+F_{33} \sigma_{3}^{2}+ \\
& 2 F_{12} \sigma_{1} \sigma_{2}+2 F_{23} \sigma_{2} \sigma_{3}+2 F_{31} \sigma_{3} \sigma_{1}+F_{44} \sigma_{4}^{2}+ \\
& F_{55} \sigma_{5}^{2}+F_{66} \sigma_{6}^{2}
\end{aligned}
$$

where $\sigma_{i}$ and $\sigma_{j}$ denote stress components, and $\sigma_{4}=\tau_{23}$, $\sigma_{5}=\tau_{31}, \sigma_{6}=\tau_{12}, t_{i j}$ denotes shear stress, and $F_{i}$ and $F_{i j}$ indicate intensity coefficient tensors, calculated by equation (4), and $i$ and $j$ belong to the collection of positive integer. 
TABLE 2: Five carbon fiber closed fixture layer angles.

\begin{tabular}{lc}
\hline Number & Laying angle \\
\hline 1 & {$\left[0^{\circ}\right]_{370}$} \\
2 & {$\left[0^{\circ} / 90^{\circ} / 0^{\circ} / 90^{\circ} / 0^{\circ}\right]_{375}$} \\
3 & {$\left[-45^{\circ} / 0^{\circ} / 45^{\circ} / 90^{\circ} /-45^{\circ} / 0^{\circ} / 45^{\circ} / 90^{\circ} /-45^{\circ} / 0^{\circ} / 45^{\circ} / 90^{\circ} /-45^{\circ} / 0^{\circ} / 45^{\circ} / 90^{\circ} /-45^{\circ} / 45^{\circ} / 0^{\circ} / 45^{\circ} /-45^{\circ} / 90^{\circ} / 45^{\circ} / 0^{\circ} /-45^{\circ} / 90^{\circ} / 45^{\circ} / 0^{\circ} /-45^{\circ} / 90^{\circ} / 45^{\circ} / 0^{\circ} /-45^{\circ} /\right.$} \\
& $\left.90^{\circ} / 45^{\circ}\right]_{5 S}$ \\
\hline
\end{tabular}

Note: taking the second ply angle as an example, number ' $37^{\prime}$ ' indicates that there are 37 ply groups, and ' $s$ ' indicates that all plies are symmetrical.

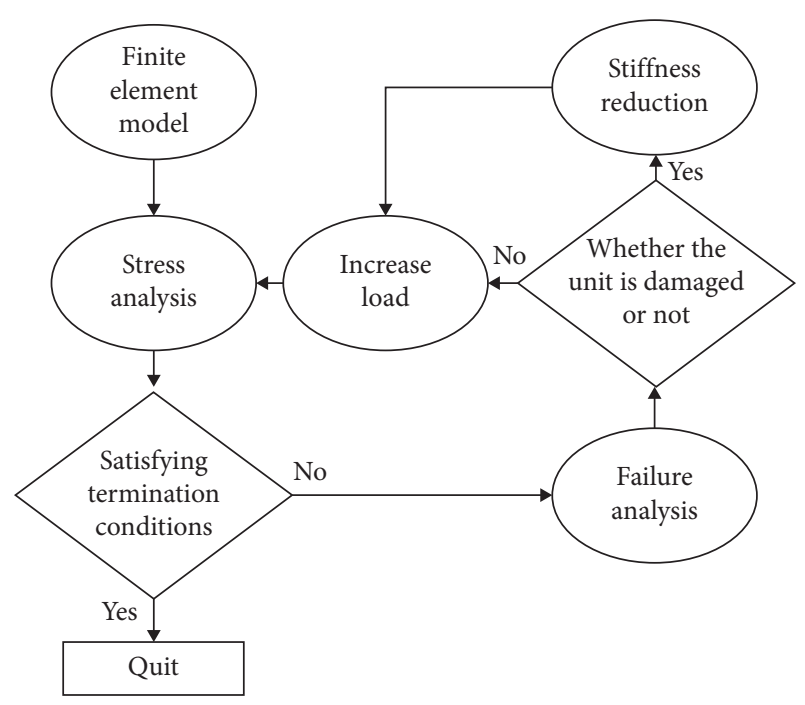

Figure 7: Progressive damage analysis flowchart.

$$
\left\{\begin{array}{l}
F_{1}=\frac{1}{A_{t}}-\frac{1}{A_{c}}, F_{11}=\frac{1}{A_{t} A_{c}}, \\
F_{2}=\frac{1}{B_{t}}-\frac{1}{B_{c}}, F_{22}=\frac{1}{B_{t} B_{c}}, \\
F_{3}=\frac{1}{C_{t}}-\frac{1}{C_{c}}, F_{22}=\frac{1}{C_{t} C_{c}}, \\
F_{44}=\frac{1}{S_{23}^{2}}, F_{55}=\frac{1}{S_{13}^{2}}, F_{66}=\frac{1}{S_{12}^{2}}, \\
F_{12}=-\left(\frac{1}{2 \sqrt{F_{11} F_{22}}}\right), F_{23}=-\left(\frac{1}{2 \sqrt{F_{22} F_{33}}}\right), \\
F_{13}=-\left(\frac{1}{2 \sqrt{F_{11} F_{33}}}\right),
\end{array}\right.
$$

where $A_{t}$ and $A_{c}$ denote the tensile strength and the compressive strength of the carbon fiber composite material in the 1 direction, $B_{t}$ and $B_{c}$ denote the tensile strength and the compressive strength in the 2 direction, and $C_{t}$ and $C_{c}$ denote the tensile strength and the compressive strength in the 3 direction. $S_{23}, S_{13}$, and $S_{12}$ denote the shear strength on the in-plane and out-of-plane, respectively.

In Tsai-Wu tensor theory, the failure judgment is to substitute the calculated element stress into formula (3). If
$W \geq 1$, the element is judged to be damaged; otherwise, the destruction does not occur.

3.3. Stiffness Reduction. If the destruction occurs, the stiffness of the element needs to be reduced. Changing material properties of the damaged element is an efficient way to reduce the stiffness. Therefore, the material performance degradation method is adopted as shown in Table 3. $E_{i i}$ represents the elastic modulus, $G_{i j}$ and $v_{i j}$ represent the shear modulus and Poisson's ratio, and $E_{i i}{ }^{\prime}, G_{i j}{ }^{\prime}$, and $v_{i j}^{\prime}$ represent the modulus of elasticity, front modulus, and Poisson's ratio of the carbon fiber composite material after failure, respectively. Here, $i$ and $j$ belong to the collection of positive integer.

During calculation, because the material property of the element is not set to 0 , the elastic modulus and shear modulus of the element degenerate to 0.0001 and Poisson ratio degenerates to 0.01 when tensile or compressive failure occurs in the direction of 1 .

\subsection{Finite Element Modeling of the Carbon Fiber Closed} Fixture. The mechanical properties of carbon fiber closed fixture were analyzed by using the finite element progressive damage strength analysis method. Based on ABAQUS finite element software and USDFLD user subroutine, the strength criterion and stiffness reduction method were compiled, and the mechanical properties of three kinds of carbon fiber closed fixtures with different ply angles were analyzed.

Because the front clamps and the rear clamps have the same force and dimensions, only the analysis of the front or rear is conducted. The analysis of the mechanical properties of the front carbon fiber clamps is to be carried out.

According to the progressive damage strength analysis method, the stiffness of T700/T009 carbon fiber composite can be reduced according to the material performance degradation method. Figure 8 shows the stiffness changes (part) of mechanical parameters of T700/T009 carbon fiber composite under various failure modes written by ABAQUS program input window. In Figure 8, there are nine mechanical parameters and six field variables. In the first nine lines, mechanical parameters of T700/T009 carbon fiber composite are shown, and in the last six lines field variable parameters are listed. The first line expresses that the material has not been damaged, and the field variables are all zero. The second line indicates that the material is damaged in direction 1 . The material mechanical parameters are the parameters after stiffness reduction, and the first field variable is 1 . By analogy, the corresponding field variable will change from 0 to 1 when there is tension compression failure or shear failure in a certain direction. Carbon fiber materials 
TABLe 3: Composite material stiffness reduction.

Failure modes

1-directional tensile or compressive failure

2-directional tensile or compressive failure

3-directional tensile or compressive failure

1-2 in-plane shear failure

1-3 in-plane shear failure

2-3 in-plane shear failure
Material stiffness reduction method

$E_{11}=E_{22}=E_{33}=0$

$G_{12}=G_{13}=G_{23}=0$

$v_{12}=v_{13}=v_{23}=0$

$E_{22}^{\prime}=0.01 E_{22}, G_{12}^{\prime}=0.2 G_{12}$

$G_{13}^{\prime}=0.2 G_{13}, v_{12}^{\prime}=0.01 v_{12}$

$v_{23}^{\prime}=0.01 v_{23}$

$E_{33}{ }^{\prime}=0.01 E_{33}, G_{12}{ }^{\prime}=0.2 G_{12}$

$G_{13}^{\prime}=0.2 G_{13}, v_{13}^{\prime}=0.01 v_{13}$

$v_{23}^{\prime}=0.01 v_{23}$

$E_{22}^{\prime}=0.01 E_{22}, G_{12}^{\prime}=0.01 G_{12}$

$v_{12}^{\prime}=0.01 v_{12}, v_{23}^{\prime}=0.01 v_{23}$

$E_{33}^{\prime}=0.01 E_{33}, G_{13}^{\prime}=0.01 G_{13}$

$v_{13}^{\prime}=0.01 v_{13}, v_{23}^{\prime}=0.01 v_{23}$

$E_{22}^{\prime}=0.01 E_{22}, E_{33}^{\prime}=0.01 E_{33}$

$G_{12}^{\prime}=0.01 G_{12}, G_{13}^{\prime}=0.01 G_{13}$

$G_{23}^{\prime}=0.01 G_{23}, v_{12}^{\prime}=0.01 v_{12}$

$v_{13}^{\prime}=0.01 v_{13}, v_{23}^{\prime}=0.01 v_{23}$

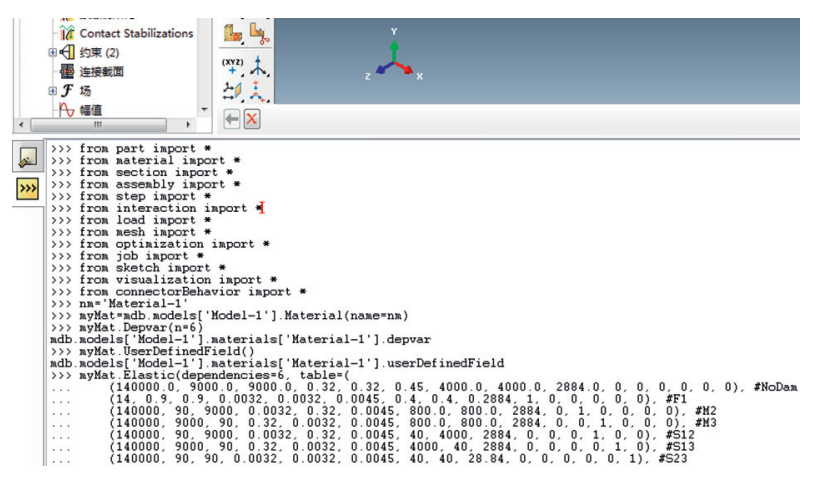

Figure 8: Stiffness and field variation of mechanical parameters of T700/T009 carbon fiber composites.

have three directions and three shear planes. There are 64 kinds of failure combinations, so there are 64 kinds of field variables.

In the process of progressive damage strength analysis, every element of the finite element model needs to be reassigned with material properties, which are to select the material mechanical parameters according to the combination form of six field variable parameters. The six field variables of the failure mode of the marking unit are controlled in real time by the USDFLD user subroutine.

The user subroutine USDFLD of ABAQUS is written in Fortran language. In the progressive damage strength analysis, the USDFLD user subroutine of ABAQUS is to calculate the 12 coefficients of Tsai-Wu strength criterion according to the failure strength parameters of T700/T009 carbon fiber composite, then to substitute the calculated element stress and 12 coefficient values into Tsai-Wu strength criterion formula to judge the material failure, and finally to update the element according to whether the failure occurs or not and the failure mode field variables of material properties and state variables of USDFLD user subroutine.

According to the stress characteristics, the inner cavity of the finite element model of carbon fiber closed fixture is set as fixed; that is, the degree of freedom in six directions is set as zero. The ends of the two wings of the finite element model are coupled to RP-1 and RP-2, respectively, and a displacement load in the thickness direction is applied to these two points to simulate the loading of the screw rod.

The loading and the constraints of finite element model of the carbon fiber closed fixture are shown in Figure 9. Finally, according to the progressive damage strength analysis method, the finite element calculation of carbon fiber closed fixture is carried out.

\section{Analysis of Simulation Results of the Carbon Fiber Closed Fixture}

Using the progressive damage strength analysis method, the failure stress cloud diagrams of the carbon fiber closed clamps with three traditional type lay angles are obtained as shown, respectively, in Figure 10. The failure positions of the three types are at the ends of the two wings, and shear failures occur.

The displacement-load curves of three type fixtures are shown in Figure 11. After undergoing elastic deformation, the three types of carbon fiber closed clamps start to enter plastic deformation from the inflection points such as A1, A2, and A3, and the carbon fiber closed clamps have been destroyed. While at the inflection points such as B1, B2, and B3, the three types of carbon fiber closed fixtures, respectively, reach the maximum bearing load, and then the fixtures are completely destroyed, and the curve decreases linearly. The values of $\mathrm{A} i$ and $\mathrm{B} i$ in the curve are shown in Table 4, where the stiffness in the elastic stage is calculated by equation (5). Here, $i$ belongs to the collection of positive integer. 


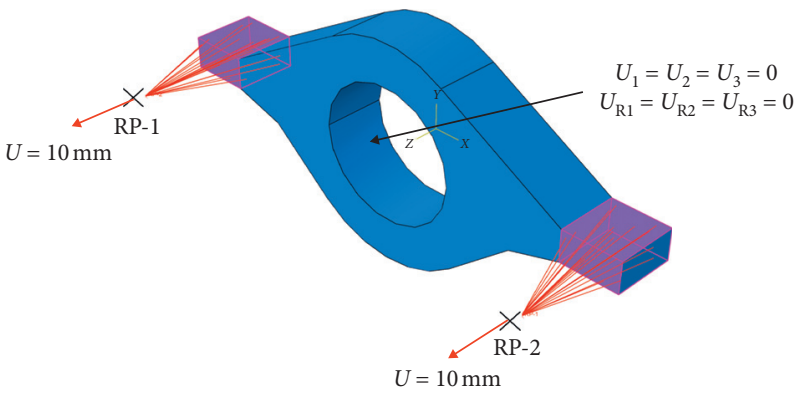

FIGURE 9: Coupling diagram of the carbon fiber closed fixture with finite element model constraints.

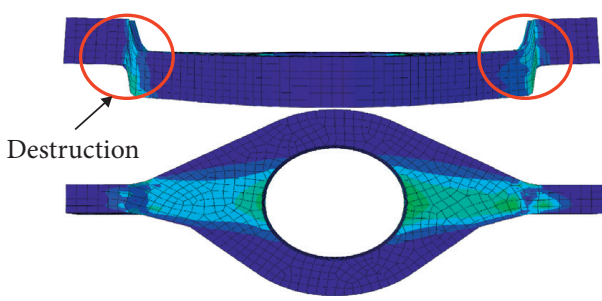

(a)

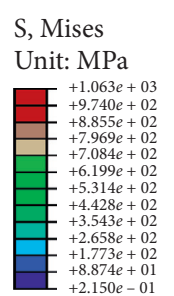

$+8.874 e+01$
$+2.150 e-01$

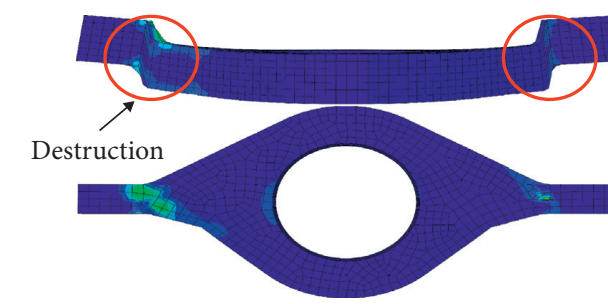

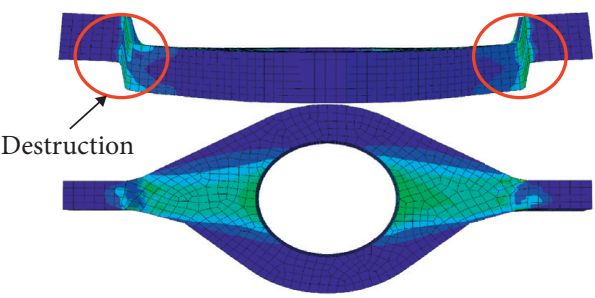

(b)
S, Mises

Unit: $\mathrm{MPa}$

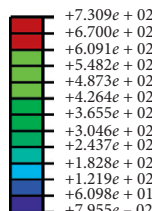

(c)

S, Mises Unit: $\mathrm{MPa}$

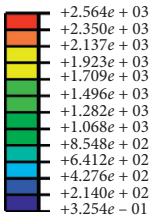

FIgURE 10: Failure stress cloud diagram of three carbon fiber closed clamps with different lay angles: (a) the first layer angle, (b) the second layer angle, and (c) the third layer angle.

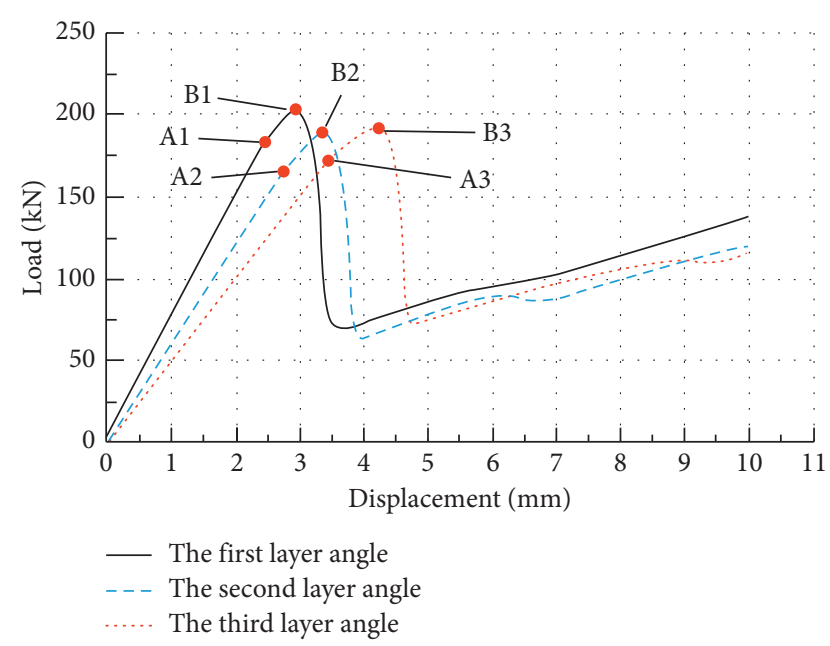

FIGURE 11: Displacement-load curve of carbon fiber closed fixtures with 3 different ply angles.

$$
k=\frac{P}{\delta}
$$

TABLE 4: Carbon fiber closed fixture data sheet with 3 different ply angles.

\begin{tabular}{lccc}
\hline Layer angle & $\mathrm{A} i(\mathrm{kN})$ & $\mathrm{B} i(\mathrm{kN})$ & Elastic stage stiffness $(\mathrm{kN} / \mathrm{mm})$ \\
\hline 1st layer & 182.52 & 203.21 & 74.79 \\
2nd layer & 164.75 & 188.74 & 57.78 \\
3rd layer & 175.71 & 191.07 & 49.48
\end{tabular}

Stiffness calculation formula is as follows:

where $k$ denotes stiffness, $P$ denotes the constant force acting on the structure, and $\delta$ denotes the displacement caused by the force.

In Table 4 , the rigidity of the $0^{\circ}$ ply (the first layer angle) is the largest, and the maximum bearing load $(\mathrm{B} i)$ is also the largest, indicating that the $0^{\circ}$ ply is more able to bear bending moment and shear than other ply angle carbon fiber closed fixtures. However, the maximum bearing load is only $203.21 \mathrm{kN}$, which is less than the maximum bearing load requirement, namely, $240 \mathrm{kN}$, resulting in that the strength requirements is not met. Therefore, the new layer structure with befitting ply angle arises. In the next section, a carbon fiber layup structure with an outer cladding layer is provided. 


\section{Improvement of the Layer Structure of the Carbon Fiber Closed Fixture}

In Section 4, the shear capacity of the ends of the two wings of the carbon fiber closed fixture is proved to be insufficient for the design. Two types of cladding structure of outer cladding angle are analyzed to select a strengthened structure.

5.1. Outer Cladding Layer Structure. To improve the failure strength of the carbon fiber closed fixture, the way is to strengthen the shear resistance of the ends of both wings. Carbon fiber outer cladding structure is shown in Figure 12, where $\theta_{i}$ indicates the lay angle. The function of this type structure is similar to that of I-beam [8]. The upper and lower horizontal layers are similar to the webs of I-beams, which is to increase the bending strength and shear strength of the whole structure. Here, $i$ belongs to the collection of positive integer.

5.2. Analysis of Simulation Results. The thickness of the outer layer is $14 \mathrm{~mm}$, known from the total thickness of the carbon fiber closed clamp which is $74 \mathrm{~mm}$, and the thickness of the inner layer is $46 \mathrm{~mm}$. As mentioned above, the carbon fiber closed fixture with $0^{\circ}$ ply has a better bending resistance; therefore, the inner ply angle is set as $0^{\circ}$ ply, and the outer ply angle is shown in Table 5.

After conducting the finite element calculations on two carbon fiber closed fixtures with different outer cladding angles, the broken-ring stress cloud image is obtained as shown in Figure 13.

The failure position of the first type of carbon fiber closed clamp with the outer cladding angle is the same as that without the outer cladding structure, and both of them appear at the ends of the two wings and are shear failures. The second type of carbon fiber closed clamp with outer cladding angle breaks around the inner cavity, with compression failure on the front and tensile failure on the back. The overall structure is a bending failure.

Figure 14 shows the displacement-load curves of two kinds of carbon fiber closed clamps with different cladding angles. The first type of carbon fiber closed clamp with outer cladding angle enters plastic deformation from the inflection point $\mathrm{A} 1$. When the $\mathrm{B} 1$ inflection point is reached, the fixture has the maximum load. After that, the clamp is completely destroyed and the curve drops straight. And the inflection point of the second type of carbon fiber closed clamp with outer cladding angle entering plastic deformation is A2. At the B2 inflection point, there is the maximum load. After that, the clamp is not completely destroyed, and the curve does not drop straight. The values of $\mathrm{A} i$ and $\mathrm{B} i$ and the stiffness of the elastic stage are shown in Table 6. Here, $i$ belongs to the collection of positive integer.

From the third column of Table 6, the strength of carbon fiber closed fixture with cladding structure is higher than the one without cladding structure. The rigidity and the maximum load $(\mathrm{B} i)$ of the first kind of closed fixture are lower than those of the second kind; thus, the second kind of angle

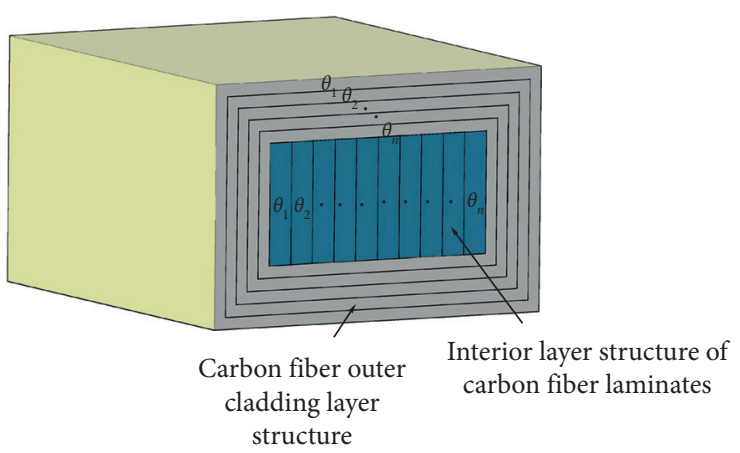

FiguRE 12: Carbon fiber outer cladding structure.

has better shear resistance. That is to say, the $\pm 45^{\circ}$ layer has a better shear resistance than the $0^{\circ}$ and $90^{\circ}$ layer. According to the failure stress cloud diagram of the fixture shown in Figure 13(b), the second overlay angle enhances the shear strength of the ends of the two wings, which makes the failure position transfer to the surrounding of the fixed area and the failure mode turns to bending failure.

The maximum bearing load of the second kind of carbon fiber closed clamp with the angle of outer cladding is $472.6 \mathrm{kN}$, which is higher than the maximum bearing load required by the carbon fiber closed clamp. Therefore, the second type of cladding structure of the outer cladding angle is used to manufacture the new clamp.

\section{Experimental Results and Analysis}

6.1. Fabrication and Test of Carbon Fiber Closed Fixtures. The carbon fiber closed clamp manufactured is shown in Figure 15, and total weight is $17.46 \mathrm{~kg}$, which is $36.46 \%$ lighter than the XWP-420 titanium alloy closed fixture.

Mechanical test is conducted on YWL-100 hydraulic horizontal tensile testing machine which contains hydraulic tensile machine and control platform, shown in Figure 16. The hydraulic tensile machine includes the front and rear chuck seats: the front chuck seat is controlled by the control platform and the rear chuck seat is manually pushed to adjust the test space in stages. The overall frame adopts steel plate welded frame structure, and the control platform adjusts the load applied by the front chuck seat and displays the test force and test curve, and automatically processes the test data according to the requirements of the test method. Due to fast response and high sensitivity, the load, displacement, and deformation of the test piece are collected in real time.

According to China power industry standard "DL/T 875-2016," the carbon fiber closed clamp and two insulators are assembled and installed on the front chuck seat and the rear chuck seat of the hydraulic tension machine, as shown in Figure 17. Then, a tensile force as $80 \mathrm{kN}$ is applied to the carbon fiber closed fixture through the control platform and kept for 5 minutes. The loading rate of the entire test is $0.221 \mathrm{kN} / \mathrm{s}$.

If no permanent deformation or damage, flexible and reliable operation, and no jamming occurs, the carbon fiber closed fixture is considered qualified. 
TABLE 5: Two outer cladding layer angles.

\begin{tabular}{lc}
\hline Number & Outer layer angle \\
\hline 1 & {$\left[0^{\circ} / 90^{\circ} / 0^{\circ} / 90^{\circ} / 0^{\circ}\right]_{23 S}$} \\
2 & {$\left[-45^{\circ} / 0^{\circ} / 45^{\circ} / 90^{\circ} /-45^{\circ} / 0^{\circ} / 45^{\circ} / 90^{\circ} /-45^{\circ} / 0^{\circ} / 45^{\circ} / 90^{\circ} /-45^{\circ} / 0^{\circ} / 45^{\circ} / 90^{\circ} / 45^{\circ} / 0^{\circ} / 45^{\circ} / 90^{\circ} / 45^{\circ} / 0^{\circ} /-45^{\circ} / 90^{\circ} / 45^{\circ} / 0^{\circ} /-45^{\circ} / 90^{\circ} / 45^{\circ} / 0^{\circ} /-45^{\circ} / 90^{\circ} / 45^{\circ} /\right.$} \\
& $\left.0^{\circ} /-45^{\circ}\right]_{\mathrm{S}}$ \\
\hline
\end{tabular}

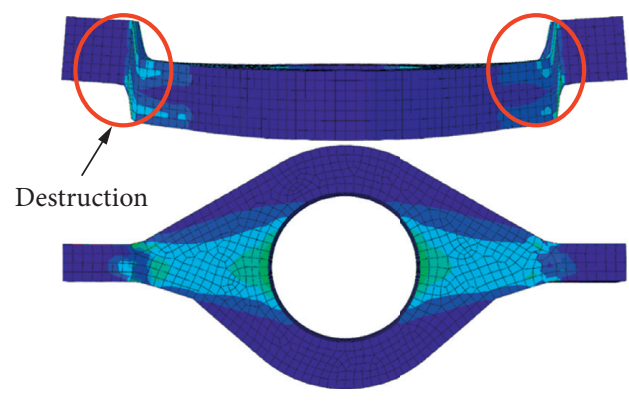

(a)
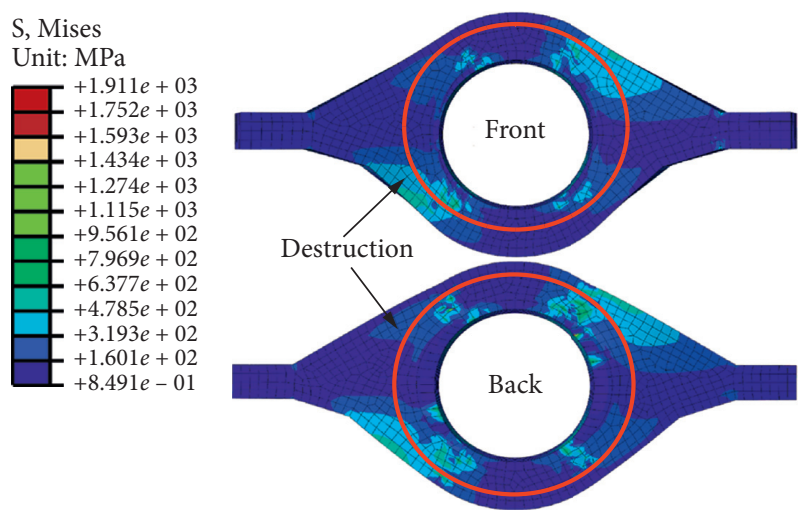

S, Mises Unit: $\mathrm{MPa}$

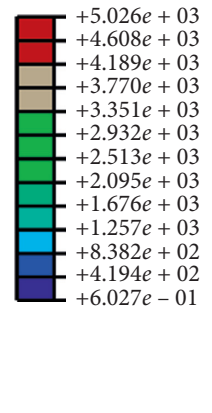

(b)

FIGURE 13: Failure stress cloud diagram of two carbon fiber closed clamps with different outer cladding angles: (a) the first angle of outer cladding; (b) the second angle of outer cladding.

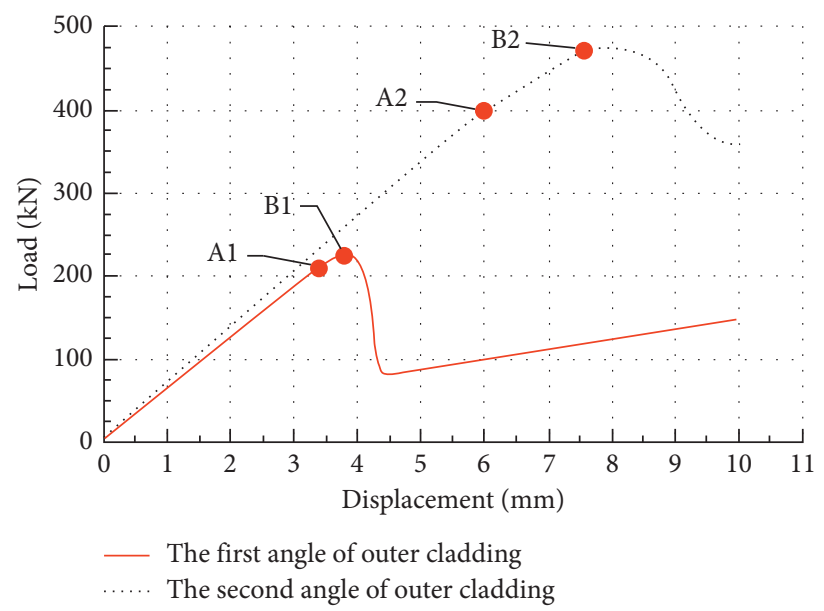

FIGURE 14: Displacement-load curves of closed carbon fiber fixtures with two outer layer angles.

TAвLE 6: Data sheet of carbon fiber closed fixtures with two outer laying angles.

\begin{tabular}{lccc}
\hline Layer angle & $\mathrm{A} i(\mathrm{kN})$ & $\mathrm{B} i(\mathrm{kN})$ & Elastic stage stiffness $(\mathrm{kN} / \mathrm{mm})$ \\
\hline 1st layer & 214.74 & 223 & 61.5 \\
2nd layer & 397.1 & 472.6 & 65.53 \\
\hline
\end{tabular}

6.2. Result Analysis. After the test, the displacement-load curve in the elastic deformation range is obtained, and the curve is compared with the displacement-load curve of the finite element calculation in the elastic deformation range with the thickness of 14-millimeter cladding layer, as shown in Figure 18. The test rigidity of carbon fiber closed fixture in elastic deformation is $60.71 \mathrm{kn} / \mathrm{mm}$, which is $4.82 \mathrm{kn} / \mathrm{mm}$ less than the rigidity calculated by finite element method, and the error between them is within $10 \%$, which meets the engineering accuracy requirements.

The result shows that the carbon fiber closed clamps in the elastic deformation range are consistent with the results of the finite element calculation. And the validity of the finite element mechanical performance analysis method and the 

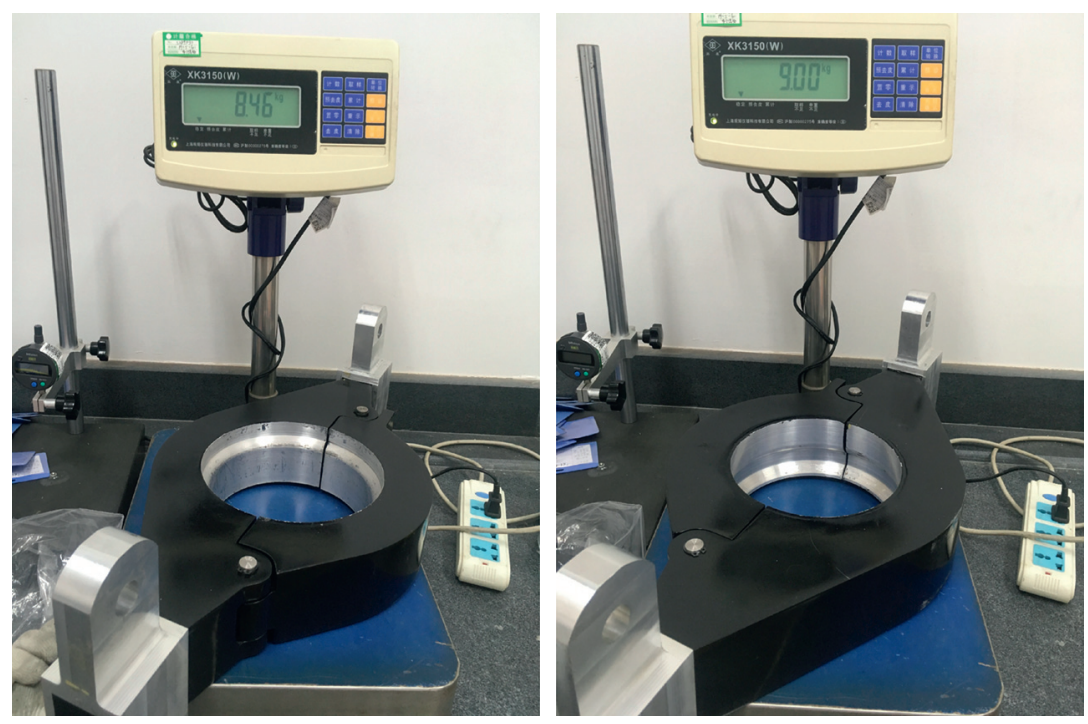

Figure 15: Carbon fiber closed fixture.

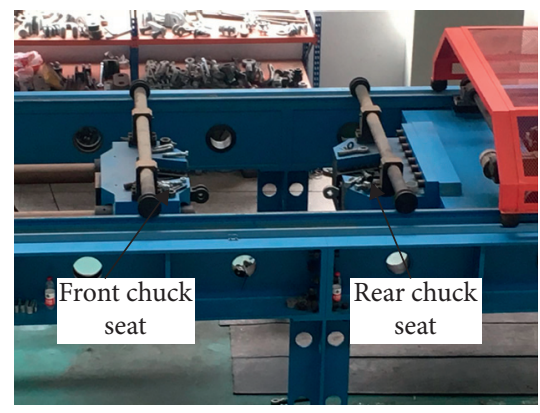

(a)

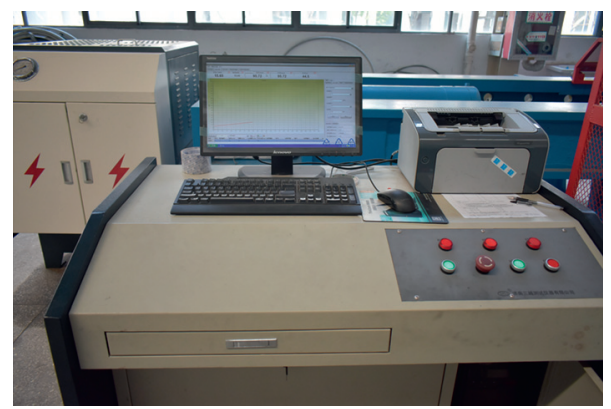

(b)

Figure 16: YWL-100 hydraulic horizontal tensile testing machine: (a) hydraulic tensile machine; (b) control platform.

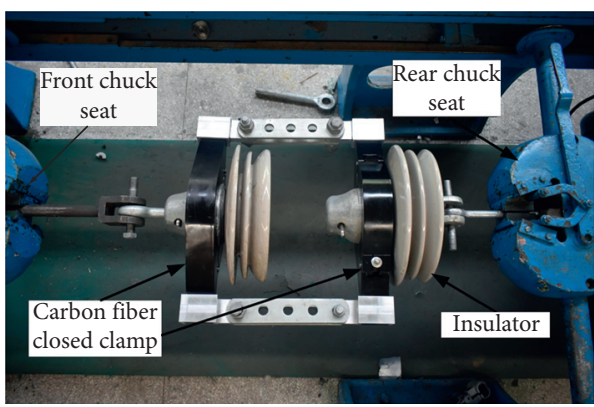

FIGURE 17: Test assembly of the closed carbon fiber fixture.

feasibility of the structural design of the carbon fiber closed clamp in the elastic deformation range are verified.

The clamp after the test is shown in Figure 19. The carbon fiber closed clamp has no signs of deformation and damage, and the bolt connection is rotated flexibly, and the connection between the metal part and carbon fiber has no falling off phenomenon; the metal part has no signs of damage, so that the new one meets the shear strength requirements of "DL/T 875-2016."

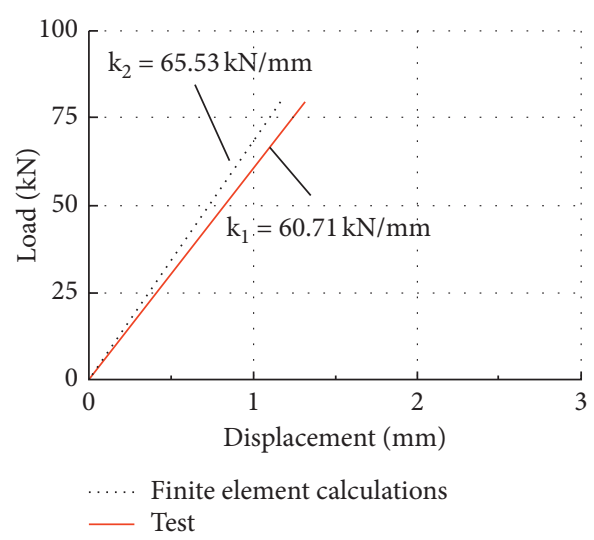

FIgURE 18: Comparison of the displacement and load curve between elastic deformation test and finite element calculation.

\section{Conclusion}

In this paper, the application of carbon fiber material is proposed for the manufacture of the closed clamp used in ultrahigh voltage transmission lines. The new carbon fiber 


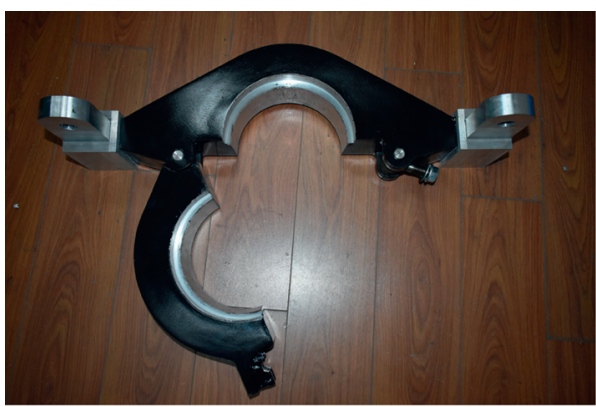

(a)

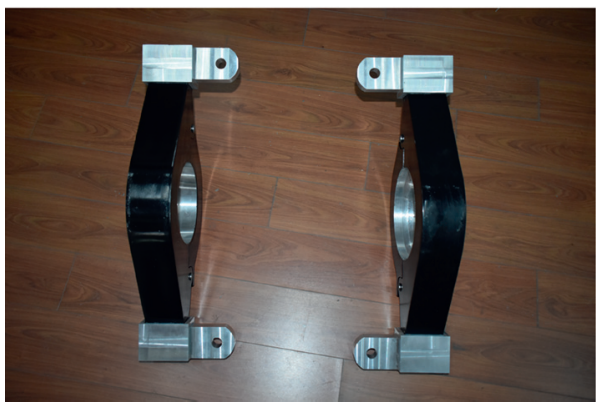

(c)

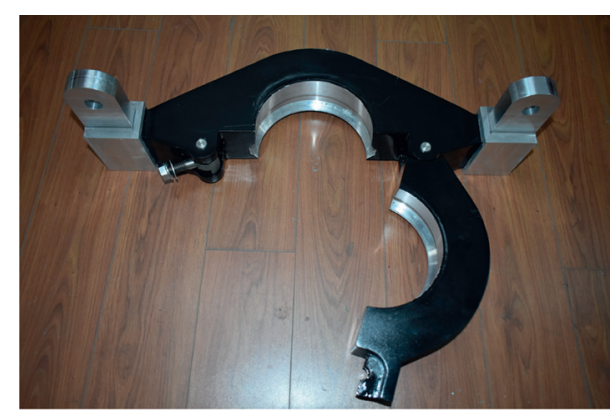

(b)

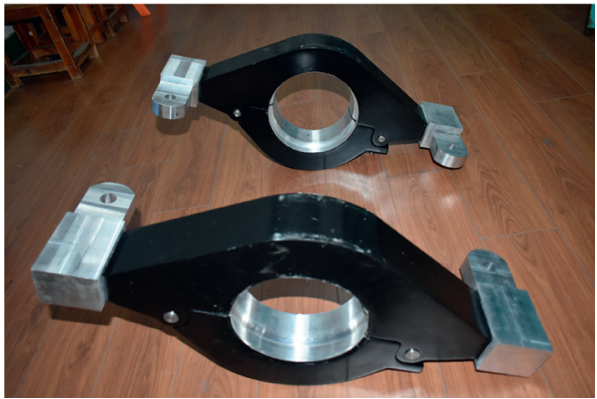

(d)

Figure 19: Carbon fiber closed clamp after test: (a) carbon fiber front fixture, (b) carbon fiber rear fixture, (c) overall top view of the carbon fiber fixture, and (d) overall side view of the carbon fiber fixture.

outer cladding structure is used to solve the problem of insufficient shear resistance of the ends of the two wings of the carbon fiber closed clamp. The manufactured equipment has passed the mechanical property test and achieved the purpose of lightweight; the weight of clamp is $36.46 \%$ lower than that of traditional titanium alloy clamp. On this basis, the destructive test and field operation test of this equipment need to be further studied.

\section{Data Availability}

The data used to support the findings of this study are available from the corresponding author upon request.

\section{Conflicts of Interest}

The authors declare that there are no conflicts of interest regarding the publication of this paper.

\section{Acknowledgments}

The authors would like to thank the National Natural Science Foundation of China (51577106) for financially supporting this research.

\section{References}

[1] J. F. Li, "Development of special insulators for inspection and repair of $1000 \mathrm{kV}$ ultra-high voltage transmission lines," Electric Power Construction, vol. 31, no. 7, pp. 33-44, 2010.

[2] J. Wang, X. Liang, and Y. Gao, "Failure analysis of decay-like fracture of composite insulator," IEEE Transactions on
Dielectrics and Electrical Insulation, vol. 21, no. 6, pp. 25032511, 2014.

[3] R. C. Petersen, "Bisphenyl-polymer/carbon-fiber-reinforced composite compared to titanium alloy bone implant," International Journal of Polymer Science, vol. 2011, Article ID 168924, 11 pages, 2011.

[4] Y. Sekii and T. Hayashi, "Measurements of reflectance and thermal emissivity of a black surface created by electrostatic flocking with carbon-fiber piles," IEEE Transactions on Dielectrics and Electrical Insulation, vol. 16, no. 3, pp. 649-654, 2009.

[5] B. Burks, D. Armentrout, and M. Kumosa, "Failure prediction analysis of an ACCC conductor subjected to thermal and mechanical stresses," IEEE Transactions on Dielectrics and Electrical Insulation, vol. 17, no. 2, pp. 588-596, 2010.

[6] M. A. Msekh, N. H. Cuong, G. Zi, P. Areias, X. Zhuang, and T. Rabczuk, "Fracture properties prediction of clay/epoxy nanocomposites with interphase zones using a phase field model," Engineering Fracture Mechanics, vol. 188, pp. 287299, 2018.

[7] H. Talebi, M. Silani, S. P. A. Bordas, P. Kerfriden, and T. Rabczuk, "A computational library for multiscale modeling of material failure," Computational Mechanics, vol. 53, no. 5, pp. 1047-1071, 2014.

[8] T. Chen, M. Qi, X. L. Gu, and Q. Q. Yu, "Flexural strength of carbon fiber reinforced polymer repaired cracked rectangular hollow section steel beams," International Journal of Polymer Science, vol. 2015, Article ID 204861, 9 pages, 2015.

[9] X. F. Tan, "Shear stability and post buckling behavior of stiffened aeronautical composite plates," Acta Materiae Compositae Sinica, vol. 35, no. 2, pp. 320-331, 2018.

[10] F. Liu, "Laminate structure design and strength analysis of I-beam composite," Mechanical Science and Technology for Aerospace Engineering, vol. 35, no. 4, pp. 641-645, 2016. 
[11] X. F. Tan, Y. T. He, and Y. Feng, "Buckling and post-buckling performance of aeronautic carbon fiber reinforced resin composite stiffened panel under compression," Journal of Aerospace Power, vol. 31, no. 10, pp. 2359-2369, 2016.

[12] J. Zang and J. Y. Zhou, "Research on optimum design of carbon fiber composite material anti-collision beam based on adaptive genetic algorithm," Journal of Machine Design, vol. 36, no. 4, pp. 87-91, 2019.

[13] F. Tao, F. Li, and F. Shao, "Reinforced reinforced foam sandwich structure in-plane compression and interface fracture performance test," Acta Materiae Compositae Sinica, vol. 35, no. 5, pp. 1123-1130, 2018.

[14] R. Huo, W. Liu, L. Wan, Y. Fang, and L. Wang, "Experimental study on sandwich bridge decks with GFRP face sheets and a foam-web core loaded under two-way bending," Advances in Materials Science and Engineering, vol. 2015, Article ID 434721, 12 pages, 2015.

[15] H. Hui, X. Y. Liu, and C. Zhang, "Research on interlayer shear properties of stitched composite foam sandwich structure," Chinese Journal of Applied Mechanics, vol. 33, no. 1, pp. 105-109, 2016.

[16] G. Reyes and S. Rangaraj, "Fracture properties of high performance carbon foam sandwich structures," Composites Part A Applied Science and Manufacturing, vol. 42, no. 1, pp. 0-7, 2011.

[17] T. Jahangiri, Q. Wang, C. L. Bak, F. F. da Silva, and H. Skouboe, "Electric stress computations for designing a novel unibody composite cross-arm using finite element method," IEEE Transactions on Dielectrics and Electrical Insulation, vol. 24, no. 6, pp. 3567-3577, 2017.

[18] A. Du, Y. Liu, H. Xin, and Y. Zuo, "Progressive damage analysis of PFRP double-lap bolted joints using explicit finite element method," Composite Structures, vol. 152, pp. 860-869, 2016.

[19] J. J. Zhou, P. H. Wen, and S. G. Wang, "Finite element analysis of a modified progressive damage model for composite laminates under low-velocity impact," Composite Structures, vol. 225, pp. 11-24, 2019.

[20] K. Shen and G. Pan, "Buckling optimization of composite cylinders for underwater vehicle applications under Tsai-Wu failure criterion constraint," Journal of Shanghai Jiaotong University (Science), vol. 24, no. 4, pp. 534-544, 2019.

[21] S. J. Deteresa and G. J. Larsen, "Reduction in the number of independent parameters for the Tsai-Wu tensor polynomial theory of strength for composite materials," Journal of Composite Materials, vol. 37, no. 19, pp. 1769-1785, 2003.

[22] X. H. Kong and Z. J. Wang, "Compressive residual strength analysis of composite laminate," Aircraft Design, vol. 34, no. 6, pp. 42-47, 2014.

[23] X. Yan and H. P. Zhang, "Discussion on design parameters of composite laminates," Journal of Donghua University(Natural Science), vol. 26, no. 5, pp. 8-11, 2000. 